\title{
Myelocyte to Leukocyte Ratio
}

National Cancer Institute

\section{Source}

National Cancer Institute. Myelocyte to Leukocyte Ratio. NCI Thesaurus. Code C64826.

The determination of the ratio of myelocytes compared to leukocytes in a blood sample.

The measurement may be expressed as a ratio or percentage. 